\title{
riccafd
}

Revista Iberoamericana de Ciencias de la Actividad Física y el Deporte

\section{ESTUDIOS ALEATORIOS SOBRE EL EFECTO DEL SENIOR FITNESS TEST EN LA TERCERA EDAD}

\section{RANDOMIZED STUDIES ON THE EFFECT OF SENIOR FITNESS TEST IN THE ELDERLY}

Gómez Figueroa, JA.1; Castineyra Mendoza, S.2; Rodríguez Cervantes, VO.3; Quintana Rivera, L.4; Chacón Rodríguez, CM.5; Rivera Girón, AJ.6; Gasperin Rodríguez, El.7; Molina Arriola, JE.8; Flores Lozano, JC. ${ }^{9}$

${ }^{1}$ Dr. Julio Alejandro Gómez Figueroa1, julgomez@uv.mx

${ }^{2}$ Santiago Castineyra Mendoa. Licenciado en Educación Física. scastineyra@uv.mx

${ }^{3}$ Víctor Osiris Rodríguez Cervantes. Licenciado en Educación Física. osirisrodriguez1190@gmail.com

${ }^{4}$ Dr. Luis Quintana Rivera. Iquintana@uv.mx

${ }^{5}$ Carlos Manuel Chacón Rodríguez. Licenciado en Educación Física. cchacon@uv.mx

6 Ángel de Jesús Rivera Girón. Licenciado en Educación Física. angelrivera02@uv.mx

${ }^{7}$ Edgar Ismael Gasperin Rodríguez. Licenciado en Educación Física. egasperin@uv.mx

${ }^{8}$ José Eduardo Molina Arreola. Licenciado en Educación Física.josemolinaef@hotmail.com

9 Julio Cesar Flores Lozano. Licenciado en Educación Física.jlozano@uv.mx

1,2,3,4,5,6,7,8,9 Facultad de Educación Física, Deporte y Recreación de la Universidad de

la Universidad Veracruzana. México

Código UNESCO: 3201.07 Geriatría

Clasificación del Consejo de Europa: 4. Educación Física y Deporte Comparado

DOI:http://dx.doi.org/10.24310/riccafd.2018.v7i3.5540

\section{RESUMEN}

Introducción: Se ha observado una relación con un buen estado físico de las personas de la tercera edad con el programa Senior Fitness Test.

Objetivo: Analizar estudios aleatorizados (EA) en personas de la tercera edad, con una intervención entre 10 y 12 semanas, que se analicen cada una de las pruebas de la batería SFT: Chair Stand, Arm Curl, 6- Minute Walk, 2- Minute Step, Chair-Sit and Reach, Back Scratch y 8-Foot up- And-Go.

Metodología: Se realizo una búsqueda en las bases de datos:PubMed, Dialnet, Google Shocolar, Elsevier y Scopus, de EA, del 2000 al 2017, la estrategia de búsqueda fue "Senior Fitness Test, Adulto Mayor y Actividad Física", se encontraron 4 estudios con condiciones ideales para el análisis.

Resultados: En el primer estudio existió diferencia significativa en solo una de sus pruebas $(\mathbf{p}=\mathbf{0 . 0 0 1})$ en un segundo estudio, dos pruebas arrojaron mejoras 
en la fortaleza (sentarse y levantarse), $(\mathbf{p}=\mathbf{0 . 0 2 2})$ y en su flexibilidad $(\mathbf{p}=\mathbf{0 . 0 4 6})$, en otro estudio la fuerza en miembros inferiores fue modificada de manera positiva $(p=0.001)$ de igual manera que la flexibilidad en brazos $(p=0.001)$.

Conclusión: Los resultados indican una mejoría en ciertas capacidades físicas de las personas de la tercera edad, pero no en todas, es por ello por lo que se recomienda estudios longitudinales con mayor homogeneidad.

Palabras clave: Senior Fitness Test, Tercera Edad, Efecto.

\section{ABSTRACT}

Introduction: A relationship has been observed with a good physical condition of the elderly people with de Senior Fitness Test Program (SFT)

Objective: The aim of this study is analyzed randomized clinical trials (RCT) with elderly people, like an intervention between 10 and 12 weeks that all battery test are analyzed SFT: Chair Stand, Arm Curl, 6- Minute Walk, 2- Minute Step, ChairSit and Reach, Back Scratch y 8-Foot up- And-Go.

Methodology: A search was made in the data bases: PubMed, Dialnet, Google Shocolar, Elsevier y Scopus, from 2000 to 2017, the search strategy was "Senior Fitness Test, Elderly and Physical Activity" four papers were founded with ideals conditions for the analysis.

Results: The first study only one test show significant differences $(p=0.001)$ the second paper, two test showed improvements in the strength (sit down and get up), $(p=0.022)$ and their flexibilities $(p=0.046)$, other study the strength in lower limbs was modified in a positive way $(\mathbf{p}=\mathbf{0 . 0 0 1})$, the same way the flexibility in arms $(\mathbf{p}=0.001)$

Conclusion: The results show an improve in some physical capacities in elderly people, but not in all, for that reason is recommended longitudinal studies with major homogeneity

Keywords: Senior Fitness Test, Elderly, Effect.

\section{INTRODUCCIÓN}

En la actualidad el mundo se encuentra en un periodo de transición generacional, de acuerdo con el informe mundial sobre el envejecimiento, hay actualmente 600 millones de personas mayores de 60 años en el mundo, cifra que se duplicará para el 2025 y que ascenderá a casi 2 mil millones para 2050¹. 
Todos los términos que pueden vincularse a la tercera edad (viejo, vejez y envejecimiento), en principio hacen referencia a una condición temporal y concretamente a una forma de tener en cuenta el tiempo y sus consecuencias en el individuo, es decir, a la edad. Según el criterio cronológico, la vejez se inicia a partir de los sesenta años, de acuerdo con la definición de las Naciones Unidas, frontera que ha ido variando rápidamente en los últimos tiempos, más que en toda la historia de occidente ${ }^{2}$. Para los fines de este trabajo, tomaremos la edad de 60 años, entonces, como inicio de la etapa de adulto mayor. ${ }^{3}$

Muchos son los cambios que se manifiestan a nivel del sistema nervioso durante la tercera edad, puesto que junto al cerebro conforman el centro del control del cuerpo. La médula espinal, se ve atrofiada, lo que trae como consecuencia que funciones reguladoras autonómicas como las encargadas de controlar la temperatura corporal, la presión sanguínea y los esfínteres resulten también disminuidas con el avance de la edad, haciendo que cada vez su deterioro sea inminente. ${ }^{4}$ En el período de la vejez se manifiesta la marcha característica del anciano (marcha dubitativa), con pasos reducidos en su amplitud, asimétricos y con menor velocidad. ${ }^{5}$ Otra de las aseveraciones es que existe mayor oscilación del centro de gravedad y disminución del balanceo de brazos. A estos cambios se le agregan una disminución del equilibrio y la coordinación, el enlentecimiento de los movimientos motores hábiles y alteraciones de la velocidad de movimientos por hiper o hipocinesias, que conducen a una alta prevalencia de caídas. ${ }^{6}$

Una de las principales estrategias en este colectivo de la tercera edad en la salud pública, es la actividad física, Diversos estudios longitudinales con adultos mayores de 70 años, se ha demostrado que por cada aumento de la $S D$ de gasto energético (medido con agua doblemente marcada) en actividades cotidianas, el riesgo de mortalidad se reducía un $32 \%$ de manera independiente. ${ }^{12}$ Otro dato interesante es que las personas mayores de 40 años, con un mínimo de dos factores de riesgo crónicos, que participaron en estudios longitudinales, de 3.5 años, se encontraron que aquellas personas que realizaban menos de 30 minutos de actividad física al día, presentaron una mortalidad 2.8 veces mayor que aquellos que realizaban $+30 \mathrm{~min} / \mathrm{día}^{7}$

Es sabido que los ejercicios físicos en la tercera edad ayudan a mantener y recuperar el equilibrio físico y mental, atrasan la involución y deterioro del músculo esquelético, lo que facilita la actividad articular y previene la osteoporosis y fracturas óseas. ${ }^{8}$ Las actividades físico-deportivas influyen en el envejecimiento cardiovascular, previniendo la arteriosclerosis y mejorando la función endócrina, principalmente la suprarrenal, para la resistencia y adaptación al estrés. ${ }^{9}$

Diversos son los estudios de revisión relacionados con el deterioro físico y la edad, estos revelan que la actividad física tiene un efecto potencial al largo de toda la vida para reducir, retardar y prevenir el proceso de deterioro de la forma física. ${ }^{10}$ La evidencia científica muestra que el ejercicio físico practicado con regularidad y de manera apropiada comporta beneficios, independientemente de la edad, sexo, nivel de salud o condición física de la persona que se somete al mismo. ${ }^{11}$ 
Es por ello por lo que el ejercicio físico es utilizado ampliamente como herramienta para paliar las limitaciones que va imponiendo el proceso de envejecimiento, aunque este debe ser individualizado a las características pertinentes de la persona mayor. ${ }^{12}$ Sin embargo, es necesaria la valoración de la condición física de ésta. Para la realización de la valoración de las capacidades físicas del adulto mayor, existe la batería de Senior Fitness Test $(\mathrm{SFT})^{13}$ esta es una batería para tal valoración, y es una de las pocas que está adaptada a los mayores. ${ }^{14}$ Las personas mayores de 65 años representan el grupo social que está creciendo a mayor velocidad en el conjunto de los países desarrollados ${ }^{15}$

Esta batería fue desarrollada reconociendo la necesidad de un instrumento para evaluar la capacidad funcional del adulto mayor, entendida como la condición física para realizar actividades de la vida cotidiana de forma eficiente, segura, independiente y sin fatiga excesiva; por esta razón investigadores de la Universidad Estatal de California, Fullerton, se dieron a la tarea de desarrollar y validar una batería de pruebas de la capacidad funcional, que incluye estándares de desempeño, denominada Senior Fitness Test (SFT). Estudio en el que participaron 7,183 estadounidenses de 60 a 94 años $1 .{ }^{16}$

Es por ello, que el objetivo principal de esta revisión bibliográfica es exponer los principales estudios relacionados con la batería Senior Fitness Test, describiendo los efectos que se han presentado en cada uno de sus elementos, dando la pauta para evaluaciones con mayor precisión en el adulto mayor.

\section{METODOLOGÍA}

Para el desarrollo de esta revisión descriptiva, se analizaron diversos estudios referentes a los efectos de la batería SFT en el adulto mayor. La búsqueda bibliográfica incluyo, manuscritos en idioma español e inglés, en fechas comprendidas del 2000 al 2017. Las bases de datos utilizadas para la búsqueda aleatoria de estos artículos fueron: PubMed, Dialnet, Google Shocolar, Elsevier, Scopus. Las palabras claves y las estrategias de búsqueda fueron: Senior Fitness Test, adulto mayor, actividad física (Senior Finess Test, Physical Activity, Elderly). Para la inclusión de los manuscritos los textos deberían presentar resultados pre y post a la batería del SFT, al igual que arrojarán resultados de todos sus elementos de este test y que la población evaluada oscilara entre los 60 y 90 años. Se excluyeron todos los textos que no incluían resultados previos o posteriores a la intervención de la batería SFT.

\section{Senior Fitness Test (SFT)}

La batería SFT, diseñada por Rikli y Jones, surgió por la necesidad de crear una herramienta que nos permitiese valorar la condición física de los mayores con seguridad, así como de forma práctica. 
La SFT puede realizarse en personas con diferentes edades entre $60-90$ años y niveles de capacidad física y funcional ya que esta batería cubre un amplio rango de capacidad funcional, desde los más frágiles a la elite.

La SFT es de fácil aplicación en cuanto al equipamiento y espacio necesarios, por lo que puede realizarse fuera del laboratorio.

A continuación, vamos a realizar una descripción completa de cada uno de los test que componen la batería Senior Fitness Test incluyendo el objetivo, el procedimiento, la puntuación y normas de seguridad. ${ }^{17}$

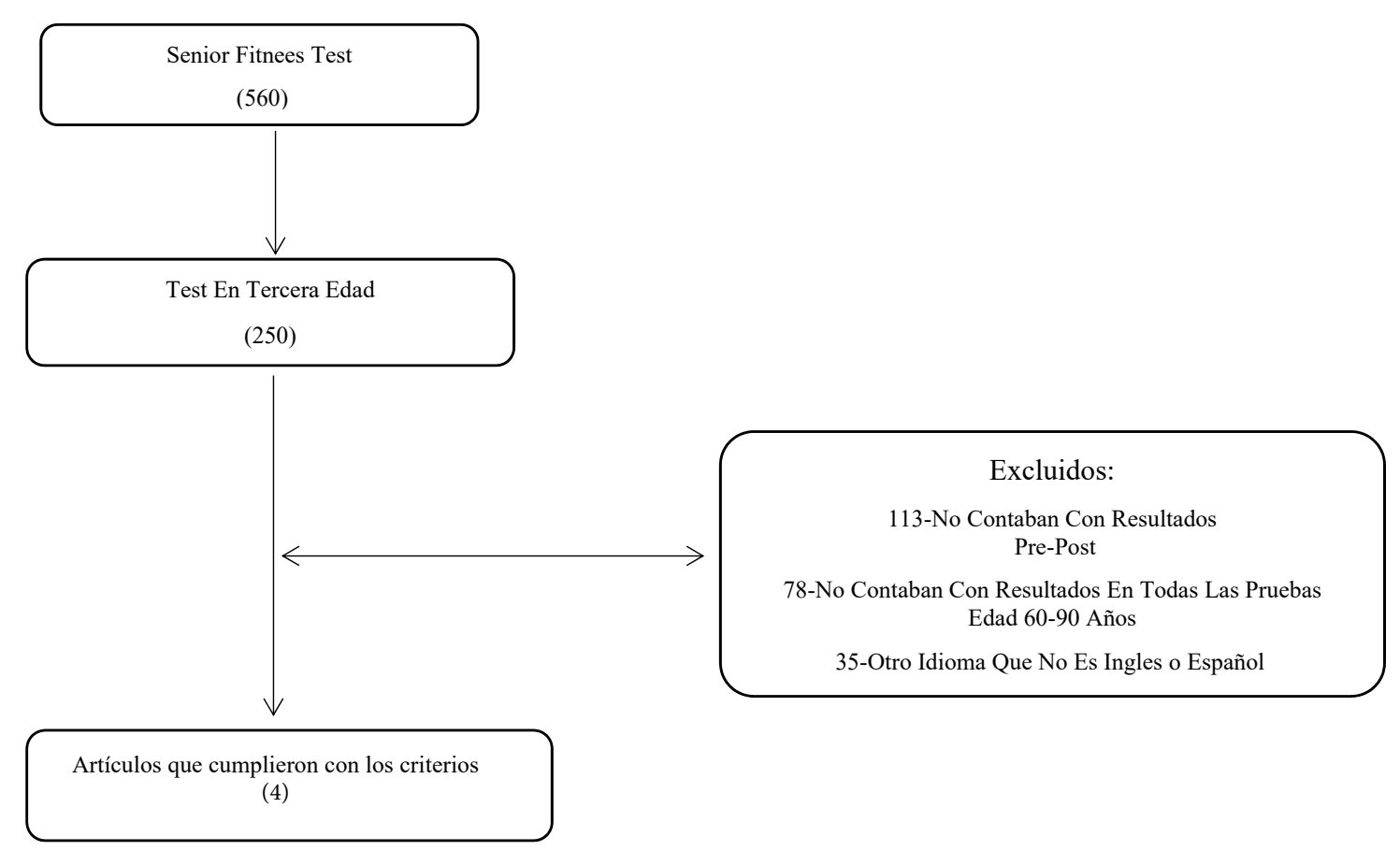

Figura 1. Diagrama de Flujo

\section{Chair Stand Test (Sentarse y levantarse de una silla)}

Objetivo: Evaluar la fuerza del tren inferior.

Procedimiento: El participante comienza sentado en el medio de la silla con la espalda recta, los pies apoyados en el suelo y los brazos cruzados en el pecho. Desde esta posición y a la señal de "ya" el participante deberá levantarse completamente y volver a la posición inicial el mayor número de veces posible durante 30 ". Tenemos que demostrar el ejercicio primero lentamente para que el participante vea la correcta ejecución del ejercicio y después a mayor velocidad para que así comprenda que el objetivo es hacerlo lo más rápido posible, pero con unos límites de seguridad. Antes de comenzar el test el participante realiza- 
rá el ejercicio uno o dos veces para asegurarnos que lo realiza correctamente.

Puntuación: Número total de veces que "se levanta y se sienta" en la silla durante 30". Si al finalizar el ejercicio el participante ha completado la mitad o más, del movimiento (levantarse y sentarse), se contará como completo. Se realiza una sola vez.

\section{Arm Curl Test (Flexiones del brazo)}

Objetivo: Evaluar la fuerza del tren superior.

Procedimiento: El participante comienza sentado en la silla con la espalda recta, los pies apoyados en el suelo y la parte dominante del cuerpo pegado al borde de la silla. Cogemos el peso con el lado dominante y lo colocamos en posición perpendicular al suelo, con la palma de la mano orientada hacia el cuerpo y el brazo extendido. Desde esta posición levantaremos el peso rotando gradualmente la muñeca (supinación) hasta completar el movimiento de flexión del brazo y quedándose la palma de la mano hacia arriba, el brazo volverá a la posición inicial realizando un movimiento de extensión completa del brazo rotando ahora la muñeca hacia el cuerpo. A la señal de "ya" el participante realizará este movimiento de forma completa el mayor número de veces posible durante 30". Primero lo realizaremos lentamente para que el participante vea la correcta ejecución del ejercicio y después más rápido para mostrar al participante el ritmo de ejecución. Para una correcta ejecución debemos mover únicamente el antebrazo y mantener fijo el brazo (pegar el codo al cuerpo nos puede ayudar a mantener esta posición)

Puntuación: Número total de veces que "se flexiona y se extiende" el brazo durante 30". Si al finalizar el ejercicio el participante ha completado la mitad o más, del movimiento (flexión y extensión del brazo), se contará como completa. Se realiza una sola vez.

\section{6- Minute Walk Test (test de caminar 6 minutos)}

Objetivo: Evaluación de la resistencia aeróbica.

Preparación: Antes de comenzar la prueba prepararemos el circuito rectangular que tendrá las siguientes medidas: (20 yardas/18,8 m) por (5 yardas/4,57m), cada extremo del circuito estará marcado por un cono y cada 5 yardas/ $4,57 \mathrm{~m}$ lo marcaremos con una línea.

Procedimiento: Se realizará una vez terminadas todas las pruebas. Saldrán de uno en uno cada 10 segundos. A la señal de "ya" el participante caminará tan rápido como le sea posible durante 6 minutos siguiendo el circuito marcado. Para contar el número de vueltas realizado el examinador dará un palillo al participante por cada vuelta realizada o lo marcará en la hoja de registro. A los 3 y a los 2 minutos se avisará del tiempo que queda para finalizar la prueba para que los participantes regulen su ritmo de prueba. Cuando pasen los 6 minutos el par- 
ticipante se apartará a la derecha y se colocará en la marca más cercana manteniéndose en movimiento elevando lentamente las piernas de forma alternativa. Puntuación: La puntuación se recogerá cuando todos los participantes hayan finalizado la prueba. Cada palillo o marca en la hoja de registro representa una vuelta (50 yardas $/ 45,7 \mathrm{~m}$ ). Para calcular la distancia total recorrida multiplicaremos el número de vueltas por 50 yardas o $45,7 \mathrm{~m}$. Se realizará un solo intento el día de la prueba, pero el día anterior todos los participantes practicarán el test para obtener el ritmo de la prueba.

\section{2- Minute Step Test (2-Minutos Marcha)}

Objetivo: Evaluación de la resistencia aeróbica.

Preparación: Antes de comenzar la prueba mediremos la altura a la que tiene que subir la rodilla el participante llevando un cordón desde la cresta ilíaca hasta la mitad de la rótula, después lo mantendremos sujeto desde la cresta ilíaca y lo doblaremos por la mitad marcando así un punto en el medio del muslo que indicará la altura de la rodilla en la marcha. Para visualizar la altura del paso transferiremos la marca del muslo a la pared para que el participante pueda tener una referencia

Procedimiento: A la señal de "ya" el participante comienza a marchar en el sitio el mayor número de veces que le sea posible durante 2 minutos. Aunque las dos rodillas deben llegar a la altura indicada, contabilizaremos el número de veces que la rodilla derecha alcanza la altura fijada. Si el participante no alcanza esta marca le pediremos que reduzca el ritmo para que la prueba sea válida sin detener el tiempo.

Puntuación: La puntuación corresponderá al número total de pasos completos (dcha.-izq.) que es capaz de realizar en 2 minutos que será el número de veces que la rodilla derecha alcanza la altura fijada. Se realizará un solo intento el día del test (el día anterior todos los participantes practicarán el test).

\section{Chair-Sit and Reach Test (Test de flexion del tronco en silla)}

Objetivo: Evaluar la flexibilidad del tren inferior (principalmente bíceps femoral)

Procedimiento: El participante se colocará sentado en el borde de la silla (el pliegue entre la parte alta de la pierna y los glúteos debería apoyarse en el borde delantero del asiento). Una pierna estará doblada y con el pie apoyado en el suelo mientras que la otra pierna estará extendida tan recta como sea posible enfrente de la cadera. Con los brazos extendidos las manos juntas y los dedos medios igualados el participante flexionará la cadera lentamente intentando alcanzar los dedos de los pies o sobrepasarlos. Si la pierna extendida comienza a flexionarse el participante volverá hacia la posición inicial hasta que la pierna vuelva a quedar totalmente extendida. El participante deberá mantener la posi- 
ción al menos por 2 segundos. El participante probará el test con ambas piernas para ver cuál es la mejor de las dos (solo se realizará el test final con la mejor de las dos). El participante realizará un breve calentamiento realizando un par de intentos con la pierna preferida.

Puntuación: El participante realizará dos intentos con la pierna preferida y el examinador registrará los dos resultados rodeando el mejor de ellos en la hoja de registro. Se mide la distancia desde la punta de los dedos de las manos hasta la parte alta del zapato. Tocar en la punta del zapato puntuará "Cero" Si los dedos de las manos no llegan a alcanzar el pie se medirá la distancia en valores negativos (-) Si los dedos de las manos sobrepasan el pie se registra la distancia en valores positivos $(+)$.

\section{Back Scratch Test (Test de juntar las manos tras la espalda)}

Objetivo: Evaluar la flexibilidad del tren superior (principalmente de hombros)

Procedimiento: El participante se colocará de pie con su mano preferida sobre el mismo hombro y con la palma hacia abajo y los dedos extendidos. Desde esta posición llevará la mano hacia la mitad de la espalda tan lejos como sea posible, manteniendo el codo arriba. El otro brazo se colocará en la espalda rodeando la cintura con la palma de la mano hacia arriba y llevándola tan lejos como sea posible, intentando que se toquen los dedos medios de ambas manos. El participante deberá practicar el test para determinar cuál es el mejor lado. Podrá realizarlo dos veces antes de comenzar con el test. Debemos comprobar que los dedos medios de una mano están orientados hacia los de la otra lo mejor posible. El examinador podrá orientar los dedos del participante (sin mover sus manos) para una correcta alineación. Los participantes no podrán cogerse los dedos y tirar de ellos.

Puntuación: El participante realizará dos intentos con el mejor lado antes de comenzar con el test y se anotará en la hoja de registro poniendo un círculo en la mejor de ellas.

Se mide la distancia entre la punta de los dedos medianos de las dos manos. Si los dedos solo se tocan puntuará "Cero" Si los dedos de las manos no llegan a tocarse se medirá la distancia en valores negativos (-) Si los dedos de las manos se solapan se registra la distancia en valores positivos (+). Siempre se mide la distancia desde la punta de los dedos de una mano a la otra independientemente de la alineación detrás de la espalda.

\section{8-Foot up- And-Go Test (Test de levantarse, caminar y volverse a sentar)}

Objetivo: Evaluar la agilidad y el equilibrio dinámico

Preparación: Colocar una silla pegada a la pared y un cono a 8 pies $(2,44$ metros), medido desde la parte posterior del cono hasta el borde anterior de la silla. 
Procedimiento: El participante se sentará en el medio de la silla manteniendo la espalda recta, los pies apoyados en el suelo y las manos sobre sus muslos. Un pie estará ligeramente adelantado respecto al otro y el tronco inclinado ligeramente hacia delante. A la señal de "ya" el participante se levantará y caminará lo más rápido que le sea posible hasta rodear el cono y volver a sentarse. El tiempo comenzará a contar desde el momento que decimos "ya" aunque el participante no haya comenzado a moverse. El tiempo parará cuando el participante se siente en la silla.

Puntuación: El examinador realizará una demostración de la prueba al participante y el participante lo realizará una vez a modo de prueba. El test se realizará dos veces y el examinador lo registrará marcando con un círculo la mejor puntuación.

\section{RESULTADOS}

El rango de edad en los estudios incluidos fue de 60 a 75 años, existiendo solo un texto que el promedio de edad superaba los 75 años. De igual manera la población en la que se aplicaron las pruebas oscilaba entre los 20 y 40 sujetos. El periodo de intervención en 3 estudios tuvo un rango de 10 a 12 semanas, solo en 1 el rango supero las 12 semanas. De los 4 estudios, 3 se realizaron en España y solo 1 en la ciudad de Cali, Colombia. El primer estudio demostró que 3 de las 6 pruebas mostraron resultados favorables, arrojando diferencias significativas, estas fueron: sentarse y levantarse de una silla, flexión del tronco en silla, levantarse caminar y volver a sentarse, las diferencias significativas fueron de p. 001. 
Tabla 1. Características de los cuatro estudios que cumplieron con los criterios de inclusión.

\begin{tabular}{|c|c|c|c|c|c|c|c|c|}
\hline Autor & Población & Edad & $\begin{array}{l}\text { Sentarse y } \\
\text { levantarse } \\
\text { de una silla }\end{array}$ & $\begin{array}{l}\text { Flexión } \\
\text { del brazo }\end{array}$ & $\begin{array}{l}2 \text { minutos } \\
\text { de marcha }\end{array}$ & $\begin{array}{l}\text { Flexión del } \\
\text { tronco en silla }\end{array}$ & $\begin{array}{l}\text { Juntar las } \\
\text { manos tras } \\
\text { espalda }\end{array}$ & $\begin{array}{l}\text { Levantarse caminar } \\
\text { y volver a sentar }\end{array}$ \\
\hline $\begin{array}{l}\text { Figueroa Y, } \\
\text { Ortega AM, } \\
\text { Plaza CH, } \\
\text { Vergara MJ. } \\
(2013)\end{array}$ & $\begin{array}{l}35 \text { adultos. } \\
\text { Programa de } \\
12 \text { semanas }\end{array}$ & \begin{tabular}{|l}
$60-75$ \\
años
\end{tabular} & $\begin{array}{l}\text { Fuerza Pre- } \\
\text { intervención } \\
\text { (repeticiones) } \\
26,0 \\
\text { Fuerza Post- } \\
\text { intervención } \\
\text { (repeticiones) } \\
32,0 \\
\text { (p valor) } \\
0,000^{*}\end{array}$ & & $\begin{array}{l}\text { Capacidad } \\
\text { Aeróbica Pre- } \\
\text { intervención } \\
\text { (yardas) } \\
599.3 \\
\text { Capacidad } \\
\text { Aeróbica Post- } \\
\text { intervención } \\
\text { (yardas) } \\
672,6 \\
\text { (P valor) 0,000* }\end{array}$ & $\begin{array}{l}\text { Flexibilidad Pre- } \\
\text { intervención } \\
\text { (pulgadas) } \\
7,9 \text { flexibilidad } \\
\text { Post-intervención } \\
\text { (pulgadas) } \\
9,5 \\
\text { (p valor) } \\
0,340 \text { ) }\end{array}$ & & $\begin{array}{l}\text { Equilibrio Pre- } \\
\text { intervención } \\
\text { (segundos) } \\
12,4 \\
\text { Equilibrio Post- } \\
\text { intervención } \\
\text { (segundos) } \\
10,7 \\
\text { (p valor) } \\
\mathbf{0 , 0 0 1 *}\end{array}$ \\
\hline $\begin{array}{l}\text { Vidarte J, } \\
\text { Quintero M, } \\
\text { Herazo Y., } \\
(2012)\end{array}$ & $\begin{array}{l}40 \\
\text { Adultos } \\
\text { Programa de } \\
12 \text { semanas }\end{array}$ & 60 años & \begin{tabular}{|l} 
Fuerza \\
miembros \\
inferiores \\
pre. \\
Intervención \\
$16,1 \pm 6,7$ \\
post \\
intervención \\
$14,6 \pm 3,5$ \\
(p valor) \\
$\mathbf{0 , 2 2}$
\end{tabular} & $\begin{array}{l}\text { Fuerza } \\
\text { miembros } \\
\text { superiores } \\
\text { pre } \\
\text { Intervención } \\
17,16 \\
\text { Post } \\
\text { intervención } \\
20,63 \\
\\
\text { (p valor) } \\
2,26\end{array}$ & $\begin{array}{l}\text { Resistencia } \\
\text { aeróbica } \\
\text { Pre intervención } \\
70,3 \pm 23,2 \\
\text { Post } \\
\text { intervención } \\
83,4 \pm 15,1 \\
\text { (p valor) } \mathbf{0 , 0 0 8 *}\end{array}$ & $\begin{array}{l}\text { Flexibilidad } \\
\text { tren inferior Pre } \\
\text { intervención } \\
-6,3 \pm 7,7 \\
\text { Post intervención } \\
-5,3 \pm 8,5 \\
\text { (p valor) } \\
\mathbf{0 , 4 6}\end{array}$ & $\begin{array}{l}\text { Flexibilidad } \\
\text { tren superior } \\
\text { Pre } \\
\text { intervención } \\
-8,24 \\
\text { Post } \\
\text { intervención } \\
-5,66 \\
\text { (p valor) } \\
0,09^{*}\end{array}$ & $\begin{array}{l}\text { Agilidad y equilibrio } \\
\text { pre intervención } \\
7,68 \\
\text { Post intervención } \\
7,58 \\
\text { (p valor) } 0,72\end{array}$ \\
\hline $\begin{array}{l}\text { Pérez A., et ., } \\
(2016)\end{array}$ & $\begin{array}{l}23 \\
\text { Adultos } \\
\text { Programa de } \\
10 \text { semanas }\end{array}$ & $\begin{array}{l}77,9 \\
\pm 4,2 \\
\text { años }\end{array}$ & \begin{tabular}{|l} 
Pre- al \\
programa \\
$15 \pm 1$ \\
Rep. \\
\\
Post \\
$18 \pm 1$ Rep. \\
(p. valor) \\
$\mathbf{0 , 0 0 1 *}^{*}$ \\
\end{tabular} & $\begin{array}{l}\text { Pre- al } \\
\text { programa } \\
18 \pm 1 \text { Rep. } \\
\\
\text { Post } \\
21 \pm 1 \text { Rep. } \\
\text { (p. valor) } \\
\mathbf{0 , 0 0 1 *}^{*} \\
\end{array}$ & Pre el programa & $\begin{array}{l}\text { Pre- al programa } \\
6,2 \pm 1,9(\mathrm{~cm}) \\
\\
\text { Post } \\
3,1 \pm 1,4(\mathrm{~cm}) \\
\text { (p. valor) } \\
\mathbf{0 , 0 0 4 *}\end{array}$ & \begin{tabular}{|l|} 
Pre el \\
programa \\
$10,9 \pm 1,9(\mathrm{~cm})$ \\
\\
Post \\
$6,7 \pm 1,6(\mathrm{~cm})$ \\
(p. valor) \\
$\mathbf{0 , 0 0 1}^{*}$
\end{tabular} & $\begin{array}{l}\text { Pre- al programa } \\
6,1 \pm 0,3 \text { (s) } \\
\\
\text { Post } \\
5,8 \pm 0,1 \text { (s) } \\
\text { (p. valor) } \\
\text { NS }\end{array}$ \\
\hline $\begin{array}{l}\text { Garatachea } \\
\text { N., Val R., y } \\
\text { Fancello I. } \\
\text { (2006). }\end{array}$ & \begin{tabular}{|l|}
27 \\
Adultos \\
Programa de \\
meses
\end{tabular} & \begin{tabular}{|l|}
$67 \pm$ \\
4,49 \\
años
\end{tabular} & \begin{tabular}{|l} 
Pre el \\
programa \\
16.08 Rep. \\
\\
Post \\
22.21 \\
(p. valor) \\
0.001*
\end{tabular} & $\begin{array}{l}\text { Pre-al } \\
\text { programa } \\
20.92 \text { Rep. } \\
\\
\text { Post. } \\
24.52 \text { Rep. } \\
\text { (P. valor) } \\
\text { N.S. }\end{array}$ & \begin{tabular}{|l} 
Pre el programa \\
78.24 \\
\\
Post \\
87.48 \\
(p. valor) \\
0.001*
\end{tabular} & $\begin{array}{l}\text { Pre el programa } \\
0.12 \\
\\
\text { Post } \\
3.28 \\
\text { (p. valor) } \\
0.05\end{array}$ & $\begin{array}{l}\text { Pre el } \\
\text { programa } \\
-1.50 \\
\\
\text { Post } \\
-6.47 \\
\text { (p. valor) } \\
\text { 0.001* }\end{array}$ & $\begin{array}{l}\text { Pre el programa } \\
4.68 \\
\\
\text { Post } \\
4.72 \\
\text { (p. valor) } \\
\text { N.S. }\end{array}$ \\
\hline
\end{tabular}

En el estudio realizado por Vidarte, J Quintero, M, Herazo Y., (2012). Obtuvieron una población de 40 personas de la tercera edad, cuya edad promedio fue de 60 años. Los resultados obtenidos posterior a las 12 semanas de intervención, mostraron que, el mejoramiento de las capacidades solo fue significativo en dos pruebas: resistencia aeróbica p. 0,008., y la flexibilidad en el tren superior arrojo una diferencia significativa de 0,009. A diferencia que en las pruebas restantes no demostraron ninguna mejoría en su ejecución, mostrando resultados no significativos.

En el 2016 Pérez A., y Cols. Realizar una intervención de 10 semanas con 23 sujetos, obteniendo una edad promedio de 77,9 $\pm 4,2$ años, los resultados posteriores a las semanas de intervención arrojaron que 4 pruebas de la batería SFT., existió diferencia significativa: sentarse y levantarse de una silla p. 0,001, Flexión de brazo p.0,001., flexión de tronco en silla p. 0,004., juntar las manos tras la espalda p. 0,001. Las dos pruebas restantes no mostraron diferencias significativas durante la intervención. 
En el último estudio presentado por Garatachea N., Val R., y Fancello I., (2006). La aplicación de la batería se realizó en una población de 27 sujetos con un rango de edad de $67 \pm 4,49$. Los resultados obtenidos de este estudio marcaron que al igual que en el estudio de Figuera Y., y Cols., (2013) las pruebas con diferencia significativa fueron: levantarse y sentarse en una silla p.0,001, 2 minutos de marcha 0,001 y juntar la mano tras la espalda $p, 0,001$.

\section{DISCUSIÓN}

La mayoría de los estudios de esta revisión tuvieron un programa de intervención de 10 a 12 semanas ${ }^{5,14,15}$ a diferencia de un estudio ${ }^{16}$ en el cual la intervención tuvo una duración mayor a las 12 semanas, el primer estudio ${ }^{14}$ tuvo efectos favorables en 3 de las 6 variables analizadas, por el contrario del estudio realizado por Vidarte J., (2012) en el cual sus resultados posteriores a las 12 semanas solo mostraron resultados significativos en 2 pruebas (resistencia aeróbica y flexibilidad)

Dos estudios ${ }^{5,16}$ mostraron resultados similares y significativos en las pruebas: sentarse y levantarse de una silla, juntarse las manos tras la espalda, en las demás pruebas de la batería SFT los resultados no fueron similares.

Es importante señalar que, en los estudios analizados de esta revisión, que arrojaron resultados significativos posterior al programa de intervención fueron: sentarse y levantarse, juntar las manos tras la espalda y 2 minutos de marcha, las pruebas restantes no mostraron resultados consistentes en cada uno de los documentos revisados.

La fortaleza de esta revisión sistemática reside en que solo se incluyen estudios aleatorios e indicadores sobre la calidad de los estudios, al mismo tiempo, el rango de edad, la población y el tiempo de intervención fueron homogéneos.

De toda la batería del SFT posterior al programa de intervención, como se menciono con antelación, solo algunos mostraron resultados significativos, la otra parte fue muy inconsistente. Sin embargo, se requieren mayores estudios longitudinales para obtener resultados con el menor sesgo posible, con grupos altamente homogéneos en todas sus variables. 


\section{BIBLIOGRAFÍA}

1. Vallejo, N. G., Ferrer, R. V., Jimena, I. C., \& Fernández, J. A. D. P. (2004). Valoración de la condición física funcional, mediante el Senior Fitness Test, de un grupo de personas mayores que realizan un programa de actividad física. Apunts. Educación física y deportes, 2(76), 22-26.

2. Sánchez, J. A. S., Navarro, A. L., \& Torón, L. E. (2013). Actividad física y diferencias de fitness funcional y calidad de vida en hombres mayores. Revista Internacional de Medicina y Ciencias de la Actividad Física y del Deporte, (49), 6-19.

3. Bo y Aro, F., \& Tío, A. (2014). Evaluación de la condición física en adultos mayores: desafío ineludible para una sociedad que apuesta a la calidad de vida. Revista Universitaria de la educación física y el deporte, (7), 6-16.

4. Murillo-Campos, E., \& Ureña-Bonilla, P. (2016). Personas adultas mayores como líderes transformadoras de la subcultura del centro diurno por medio de la actividad física. Revista Electrónica Educare, 20(1).

5. Pérez-López, A., Santiago, J. G., Merino, P., Sánchez, C. V., Valverde, I. A., VALVERDE, B. Á., Cerrato, D. V. (2016). Beneficios de un programa de ejercicio físico con autocargas y materiales reciclados sobre la composición corporal y la condición física en tercera edad: estudio piloto. Revista Española de Educación Física y Deportes, (413), 21-32.

6. Herrera, E., Monzó, A. P., Bartoll, O. C., \& Abella, C. P. (2017). Efectos de la actividad física sobre la salud percibida y condición física de los adultos mayores. Journal of sport and health research, 9(1), 27-40.

7. Cobo Mejía, E. A., Ochoa González, M. E., Ruiz Castillo, L. Y., Vargas Niño, D. M., Sáenz Pacheco, A. M., \& Sandoval Cuellar, C. (2016). Confiabilidad del Senior Fitness Test versión en español, para población adulta mayor en Tunja-Colombia. Arch. med. deporte, 382-386.

8. Collins, K., Rooney, B. L., Smalley, K. J., \& Havens, S. (2004). Functional fitness, disease and independence in community-Dwelling older adults in Western Wisconsin. WMJ-MADISON-, 103(1), 42-48

9. Toraman, N. F., \& Ayceman, N. (2005). Effects of six weeks of detraining on retention of functional fitness of old people after nine weeks of multicomponent training. British journal of sports medicine, 39(8), 565-568.

10. Chen, C., Liu, K., Jafari, R., \& Kehtarnavaz, N. (2014, August). Home-based senior fitness test measurement system using collaborative inertial and depth sensors. In Engineering in Medicine and Biology Society (EMBC), 2014 36th Annual International Conference of the IEEE (pp. 4135-4138). IEEE.

11. Milanović, Z., Pantelić, S., Trajković, N., Sporiš, G., Kostić, R., \& James, N. (2013). Age-related decrease in physical activity and functional fitness among elderly men and women. Clinical interventions in aging, 8, 549.

12. Correa Bautista, J. E., Gámez Martínez, E. R., Ibáñez Pinilla, M., \& Rodríguez Daza, K. D. (2011). Aptitud física en mujeres adultas mayores vinculadas a un programa 
de envejecimiento activo. Revista de la Universidad Industrial de Santander. Salud, 43(3).

13. Rikli, R. E., \& Jones, C. J. (2013). Development and validation of criterion-referenced clinically relevant fitness standards for maintaining physical independence in later years. The Gerontologist, 53(2), 255-267.

14. Figueroa, Y., Ortega, A. M., Plaza, C. H., \& Vergara, M. J. (2014). Efectos de un programa de intervención en la condición física en un grupo de adultos mayores de la ciudad de Cali en 2013. Ciencia \& Salud, 2(8), 23-28.

15. Vidarte Claros, J. A., Quintero Cruz, M. V., \& Herazo Beltrán, Y. (2012). Efectos del ejercicio físico en la condición física funcional y la estabilidad en adultos mayores. Hacia la Promoción de la Salud, 17(2), 79-90.

16. Garatachea, N., Val, R., \& Fancello, I. (2006). Evolución de la condición física funcional de un grupo de personas mayores que realizan un programa de actividad física durante 9 meses. Revista de Ciencias de la Actividad física y del Deporte, 15.

Referencias totales citadas: 16

Referencias citadas correspondientes a la Rev Ib CC Act Fis Dep: 0 